\title{
WORKFORCE Patients' perspective of physician associates in an acute medical unit within an English district general teaching hospital - a pilot survey study
}

\author{
Authors: Qazi Zaman, ${ }^{\mathrm{A}}$ Suren Yogamoorthy, ${ }^{\mathrm{B}}$ Momina Zaman ${ }^{\mathrm{C}}$ and Ragai Mohammed Fikry Ragai Fouda ${ }^{\mathrm{D}}$
}

\begin{abstract}
Physician associates (PAs) are valuable members of the medical team. However, few data are available concerning their role and contributions in acute settings from the perspective of patients. The current study explored patients' acceptance of the role of PAs in the NHS. A questionnaire was distributed to 100 patients who were under care of PAs in the acute medical unit (AMU) of George Eliot Hospital. The questionnaire explored previous exposure to PAs, satisfaction in PA's attitude and knowledge, PA value to NHS and overall satisfaction in PA profession. Fifty women (58.1\%) and 36 men (41.9\%) with a mean age of $74.75 \pm 10$ years participated in the study. Most of the survey responders stated that the attitudes, professionalism and medical knowledge of PAs were good or very good and nearly all of respondents were satisfied or very satisfied with the role of PAs in the NHS. This pilot questionnaire survey study in the AMU of an English district general teaching hospital showed that most study participants valued the role of PAs in the NHS.
\end{abstract}

KEYWORDS: Physician associates, patients perspective, acute medical unit

\section{Introduction}

The first physician assistant educational program began in 1965 in the USA in response to a perceived shortage and maldistribution of physicians. Dr Eugene Stead, chairman of the Department of Medicine at Duke University, established a 2-year programme to formally educate physician assistants. In 2002, the concept of physician assistants started to be developed in the UK through a collaborative project between the Changing Workforce Programme (CWP) and St George's University, London. In 2006, a Competence and Curriculum Framework (CCF) for UK physician assistants was developed collaboratively with the Department of Health (DH), Royal College of Physicians (RCP) and Royal

Authors: ${ }^{\text {A }}$ consultant physician, George Eliot Hospital NHS Trust, Nuneaton, UK; ${ }^{B}$ physician associate, George Eliot Hospital NHS Trust, Nuneaton, UK; ${ }^{C}$ medical student, Ovidius University of Constanta, Constanta, Romania; ${ }^{\text {D }}$ clinical educational fellow, George Eliot Hospital NHS Trust, Nuneaton, UK
College of General Practitioners (RCGP). The first formal physician assistant programs in the UK were opened at the Universities of Birmingham and St George's University London in 2008. The CCF defined the role, scope of practice and standards of education of physician assistants in the UK. In 2015, physician assistants changed their name to physician associates (PAs) to greater reflect their developing role. In 2016, PAs attained faculty status within the RCP and held their inaugural conference at the RCP. The number of PAs in the UK is growing. At the time of the 2017 PA census, there were 218 PAs and 639 students who were eligible to respond. ${ }^{2}$ Although PAs are well established in many UK hospitals, few data are available regarding patient acceptance of the PA profession in the NHS. However, consumer assessment of their medical providers is an important aspect of effective healthcare because satisfied patients are more likely to adhere to treatment recommendations. ${ }^{3}$

Measured patient satisfaction scores are based on patient experiences that occur before, during and after a care visit, with various levels of expectation. ${ }^{4}$ Therefore, patient satisfaction varies from patient experience in that it is a judgement formed by the patient about the accomplishment of a joint end goal. ${ }^{5}$ The broader concept of 'patient experience' is defined by the Beryl Institute as the 'sum of all interactions, shaped by an organization's culture that influences patient perceptions across the continuum of care. 6 Previous studies explored patient experience of consultations with PAs in primary care, but data regarding their experience with PAs in acute medical settings are rare. ${ }^{7}$ Few data are available in relation to PAs in acute medical units (AMU) from the patient perspective. Therefore, the aim of the current study was to use a survey to evaluate impressions of a group of patients in the AMU of a medium-sized English district general teaching hospital. The survey assessed PAs and the degree of patient satisfaction with the role of PAs in the AMU.

\section{Methodology}

\section{Study setting}

\section{George Eliot hospital}

The current pilot survey study was carried out within George Eliot hospital (GEH) AMU. GEH is an integrated acute, community and primary care service provider comprising the hospital in Nuneaton and several community services. GEH serves a population of 290,000 from Nuneaton and Bedworth, North Warwickshire, 
South West Leicestershire and Northern Coventry. It has 350 beds and provides services for several medical specialties and subspecialties in addition to a 24 -hour accident and emergency (A\&E) service. There are 65,000 A\&E attendances and 19,000 emergency admissions to GEH annually.

\section{GEHAMU}

The AMU at the GEH has 41 beds (eight of which are monitored) and six side-rooms. It has an ambulatory care unit, with seating for up to 10 patients, and a well-equipped and dedicated procedure room.

\section{GEH PAs}

GEH was one of the first hospital trusts in the UK to exhibit interest in involving the newly emerging PA profession into their multidisciplinary team. The trust was an early adopter of PAs, recognising the value and potential of this new profession, and they started recruiting PAs in 2010. GEH currently has more than 10 qualified PAs and more student PA trainees across various specialties. This includes general medical wards, AMU and various specialties at GEH, including paediatrics and orthopaedics. PAs work under the supervision of doctors to support the diagnosis and management of a large number of patients, thereby ensuring the high quality and continuity of care. PAs at GEH have formed a great bond with other members of the team by being a valuable addition to the multidisciplinary team.

PAs in GEH (AMU) work under the supervision of consultants as part of a multidisciplinary team; they have direct contact with patients, taking medical histories, and carrying out physical examinations and clinical procedures, such as blood taking and cannulation. PAs are trained to develop management plans with their consultants, to analyse test results and to write electronic discharge summaries. Currently on AMU, there is also a PA who is trained in the care of older patients and who works alongside the acute medical team. This PA works alongside the doctors, nursing staff, pharmacists, social workers, occupational therapists and physiotherapists to allow implementation of comprehensive geriatric assessments for frail and vulnerable patients. During this study, because of the limited numbers of doctors on AMU, PAs took on extra responsibilities and stayed longer hours, explaining to patients the details of their conditions and its treatments and involving them in decisions about their care.

\section{Study participants}

Adult patients admitted to the GEH AMU from 1 October 2017 to 15 February 2018 were invited to participate in a survey study. Patients must have been under the medical care of PAs, supervised by consultants, for a period of at least $48 \mathrm{~h}$, to participate in the study. Patients who showed interest in participating in the study filled out a questionnaire themselves or with the help of family members or of a group of volunteering medical students. Patients in AMU who had not been under the medical care of PAs for $48 \mathrm{~h}$ or those with delirium, cognitive problems, dementia or considered to be critically ill at the time of the survey were not included in the study.

\section{Survey questionnaire}

The questionnaire used was created to cover most of the domains defined as critical to a 'good' patient experience by the NHS, which included respect, information giving, communication, physical comfort and emotional support. ${ }^{8}$

The content of the questionnaire used in the current study was developed using ideas that had emerged from interviews conducted with patients from the GEH AMU in the preceding 12 months. All efforts were made to ensure a patient-centred questionnaire and to avoid any ambiguities or lack of clarity within the questionnaire. The survey questionnaire focused on patients' experiences with PAs in the AMU at GEH. The survey comprised 17 questions, exploring whether the patient had previous exposure to PAs, and current experience with PAs regarding their attitude, professionalism and medical knowledge. The next set of questions covered patients' confidence with PAs and the value of their role to the NHS. Finally, patients were asked about their overall satisfaction with the role of the PA profession, rated on a scale of $1-5$. There was an option for the respondents to add further comments and suggestions about how to improve PA performance in a free-text box. The ethical approval committee deemed this study exempt from ethical approval.

\section{Data analysis}

Data relating to the demographic characteristics of the study participants, in addition to that obtained from the questionnaire, were anonymised and entered into a computerised database. These data were statistically analysed in terms of mean \pm standard deviation ( $\pm \mathrm{SD}$ ), frequencies (number of participants) and percentages when appropriate. These analyses were performed using Microsoft Excel 2016 and SPSS version 21 for Microsoft Windows.

\section{Results}

\section{Study participants}

The questionnaire was distributed to 100 adult patients on GEH AMU who fulfilled the inclusion criteria previously described in the methodology. Of those asked, $86 \%$ responded to the questionnaire.

\section{Demographic data}

Of the 86 participants, 50 were female $(58.1 \%)$ and 36 were male $(41.9 \%)$. Their mean age was $74.75 \pm 10$ years.

\section{Questionnaire data}

\section{Patients' previous exposure to PAs}

Of the survey participants, $~ 80 \%(65 / 85)$ mentioned that they had not been under the medical care of a PA before their current admission to the GEH AMU. Around 10\% (9/86) of the respondents had previously been under the care of a PA. Around 10\% (9/86) did not answer the question (Table 1).

\section{PAs from the patients' perspective}

Of the survey participants, $70-100 \%$ stated that the attitudes, professionalism and medical knowledge of the PAs were either good or very good (Table 1). 


\begin{tabular}{|c|c|c|}
\hline Patient survey question & $\begin{array}{l}\text { Percentage } \\
\text { of patients } \\
\text { who replied } \\
\text { with yes / very } \\
\text { good /good }\end{array}$ & $\begin{array}{l}\text { Percentage of } \\
\text { patients who } \\
\text { replied with } \\
\text { no / satisfactory / } \\
\text { poor }\end{array}$ \\
\hline $\begin{array}{l}\text { Have you been taken care } \\
\text { of by a PA in the past? }\end{array}$ & $11 \%$ & $79 \%$ \\
\hline $\begin{array}{l}\text { How good was the PA at } \\
\text { being polite? / How good } \\
\text { was the PA at making you } \\
\text { feel at ease? }\end{array}$ & $100 \%$ & $0 \%$ \\
\hline $\begin{array}{l}\text { How good was the PA at } \\
\text { actively listening to you? }\end{array}$ & $95.43 \%$ & $4.66 \%$ \\
\hline $\begin{array}{l}\text { How good was the PA at } \\
\text { assessing your medical } \\
\text { condition? }\end{array}$ & $80.23 \%$ & $19.77 \%$ \\
\hline $\begin{array}{l}\text { How good was the PA at } \\
\text { explaining your medical } \\
\text { condition and treatment? }\end{array}$ & $79.06 \%$ & $20.96 \%$ \\
\hline $\begin{array}{l}\text { What was your opinion } \\
\text { on the PA's overall level } \\
\text { of medical knowledge? }\end{array}$ & $80.23 \%$ & $19.77 \%$ \\
\hline $\begin{array}{l}\text { How good was the PA at } \\
\text { involving you in decision } \\
\text { about management? }\end{array}$ & $75.58 \%$ & $24.42 \%$ \\
\hline
\end{tabular}

Willingness of patients to see a PA again and and their confidence in PAs

Nearly all the survey responders expressed their willingness to see a PA in the future (Table 2). Nearly all of them were confident about PAs' abilities in providing care.

\begin{tabular}{|c|c|c|}
\hline Patient survey question & $\begin{array}{l}\text { Percentage } \\
\text { of patients } \\
\text { who replied } \\
\text { with strongly } \\
\text { agree / agree }\end{array}$ & $\begin{array}{l}\text { Percentage } \\
\text { of patients } \\
\text { who replied } \\
\text { with neutral } \\
\text { / disagree }\end{array}$ \\
\hline $\mathrm{PA}$ is honest and trustworthy & $90.69 \%$ & $9.31 \%$ \\
\hline $\begin{array}{l}\text { I would be happy to see PA } \\
\text { again }\end{array}$ & $100 \%$ & $0 \%$ \\
\hline $\begin{array}{l}\text { I would recommend PA to family } \\
\text { and friends }\end{array}$ & $90.69 \%$ & $9.31 \%$ \\
\hline $\begin{array}{l}\text { PA's contribution to NHS is } \\
\text { valuable to improve quality of } \\
\text { care }\end{array}$ & $95.34 \%$ & $4.66 \%$ \\
\hline $\begin{array}{l}\text { I believe that PAs should be } \\
\text { involved in range of specialties }\end{array}$ & $90.69 \%$ & $9.31 \%$ \\
\hline
\end{tabular}

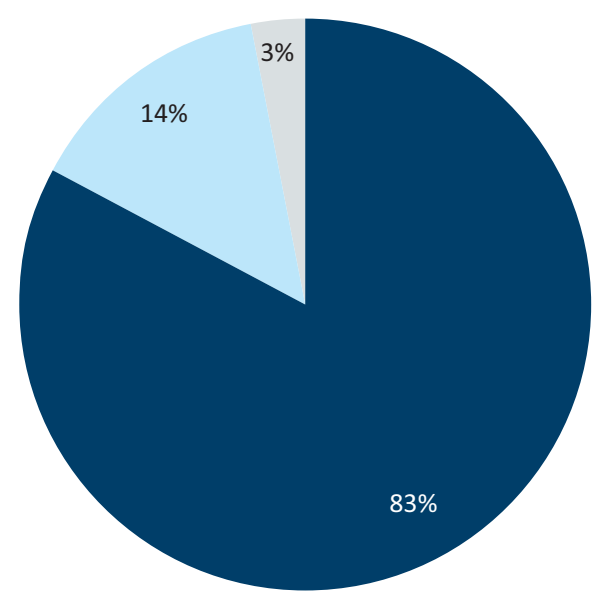

Very satisfied $5 / 5 \quad$ Satisfied $4 / 5 \quad$ Least satisfied $1 / 5$

Fig 1. Patient satisfaction with the new physician associate profession within the NHS. Pie chart showing patient responses to the question 'How satisfied are you with this new physician associate profession to NHS?'

\section{Trust and confidence in PAs}

Of the survey responders, $90-100 \%$ reported that they would recommend PAs to their family or friends and that they valued their role to the NHS (Table 2).

\section{Overall patient satisfaction with PA role in NHS}

Of the survey responders, $97 \%$ were either satisfied or very satisfied with the role of PAs in the NHS (Fig 1).

\section{Discussion}

The current pilot survey was carried out to explore the role of PAs in an AMU in a medium-sized English district general teaching hospital from the patients' perspective. In total, 86 patients participated in that study (50 women and 36 men), with a mean age of $\sim 75$ years.

Only one out of every 10 participants had previously been under the care of a PA, and most responders had a high level of trust and confidence in the PAs who treated them. Most were very satisfied with the role of PAs in the NHS. The above results showed that even those patients who had had limited previous experience with PAs were having their expectations of treatment and care met by this new profession.

Currently, there are no published UK studies exploring patient satisfaction with the role of PAs in AMUs, although a few studies exploring patient satisfaction with the PA role in general practice have been published. A US national survey study that involved 150,000 Medicare beneficiaries compared patients' satisfaction with physicians, physician assistants and nurse practitioners in general practice. It showed that, in all indices of satisfaction, PAs and nurse practitioners were rated as favourably as physicians regardless of patient sociodemographic characteristics or health status. ${ }^{9}$ Following on from this study, an interview study was done in the USA to assess patient experiences with PAs in a rural region, where they had been the only healthcare providers for the previous 2 years. The study reported mixed results, with the patients suggesting that they would sometimes prefer to see a doctor 
because of: not having confidence in the PA (not being a doctor): already having a doctor; or having a long-term condition requiring specialist care. ${ }^{10}$

In 2017, a UK telephone interview study that assessed patients' experience with PAs in six GP practices reported that, although most participants had a positive view of consultations with PAs, there were some who reported less-positive experiences. These were consultations in which the boundaries of the PA's knowledge or jurisdiction had been reached, resulting in either the transfer of the patient to the doctor or unacceptable delays in obtaining signed prescriptions. ${ }^{7}$

Despite the cross-sectional nature of the current study, which did not allow the evaluation of causality, and the lack of an in-depth study of the severity of illness of the survey participants, hospital outcome or the quality of care provided by PAs, we suggest that there are various factors that might have resulted in the high patient satisfaction scores recorded by the current study, as discussed below.

\section{Longer time spent with care provider}

A previous study showed that patients view healthcare visits as a 'time investment' service, ${ }^{11}$ so the more time spent with the provider (usually a physician or physician extender, such as a physician assistant or nurse practitioner), the higher perceived service and patient satisfaction. ${ }^{12}$ PAs spend a lot of time with patients in AMU, because they are often not rotational and do not participate in on-call duties, hence providing continuity of care. Thus, this might be one factor responsible for patients' satisfaction with the PA role in the current study.

\section{The survey participants}

Patients who are older, who achieved positive outcomes, who were sicker, or who generated higher healthcare costs were previously reported to give their physicians higher ratings. ${ }^{13}$ The current survey participants were mostly older, very sick patients, who had a favourable hospital outcome. This might be another factor responsible for their satisfaction with the role of PAs in this current study.

\section{Timing of the survey}

Studies showed that a survey completed at the end of a hospital stay provides different information compared with a survey sent out and completed a few weeks after the stay. ${ }^{14}$ Thus, completing the survey during the hospital stay might be another factor responsible for high patient satisfaction scores in the current study.

\section{Hidden factors}

Fenton et al report that the factors associated with patient satisfaction with physicians are not fully understood. ${ }^{15}$ They suggest that therapeutic responsibilities of ten require physicians to address topics that might challenge or disturb patients, including substance abuse, psychiatric comorbidity, nonadherence, and the risks of requested but discretionary tests or treatments, which could reduce patient satisfaction. ${ }^{15}$ Given that patient satisfaction correlates with the extent to which physicians fulfil patient expectations, similar issues might affect patient satisfaction with other healthcare workers, including PAs. ${ }^{16}$

\section{Strengths}

To the best of our knowledge, this is the first survey study to investigate the PA role in AMU from a patient perspective in an English NHS hospital. The high percentage of respondents on the survey $(86 \%)$ excluded bias seen in survey studies with only small percentages of respondents.

\section{Study limitations}

Despite the above strengths, the current study has several limitations.

Patients who were very unwell, confused or who had dementia were excluded from the survey study for obvious reasons. However, this might have caused a bias because only patients who were less unwell were involved in the current study.

The number of PAs working on AMU is small; therefore, the number of patients under their care included in the survey was relatively small. It is clear that a large number of survey participants is needed to obtain genuine results and exclude results due to chance.

Surveys in general have been criticised for producing bland positive responses because the data collected through interviews more frequently result in reports of negative experiences of care compared with surveys. ${ }^{17}$

The current study explored patients' perspective of PAs without evaluating these patients' experiences with other domains of healthcare in the AMU. Therefore, the findings of this pilot study are not generalisable to all NHS AMUs.

This is a pilot survey study with its own limitations that aimed to shed light on an interesting research topic. Larger future national surveys and interview studies would provide a clearer view of the acceptance of the PA role in the NHS by patients in the AMU and the factors underlying that acceptance.

\section{Conclusions}

The current pilot survey study that involved patients in the AMU in an English district general teaching hospital showed that most participants considered the attitudes, professionalism and medical knowledge of PAs to be either good or very good and indicated their willingness to see a PA again. Nearly all survey responders valued the role of PAs in the NHS.

\section{Acknowledgements}

The authors would like to thank patients, PAs, volunteered medical students and nursing staff who participated in this study.

\section{References}

1 Ross N, Parle J, Begg P, Kuhns D. The case for the physician associate. Clin Med 2012;12:200-6.

2 Royal College of Physicians. FPA census 2017. www.fparcp.co.uk/ about-fpa/fpa-census [Accessed 24 July 2018].

3 Hershey CO, Grant B]. Controlled trial of a patient-completed history questionnaire: effects on quality of documentation and patient and physician satisfaction. Am J Med Qual 2002;17:12635.

4 Berkowitz B. The patient experience and patient satisfaction: measurement of a complex dynamic. Online J Issues Nurs 2016;21:1.

5 LaVela SL, Gallan AS. Evaluation and measurement of patient experience. Patient Experience ] 2014;1:28-36. 
6 The Beryl Institute Website. Defining patient experience. www.theberylinstitute.org/?page=DefiningPatientExp [Accessed 24 July 2018].

7 Halter M, Drennan VM, Joly LM et al. Patients' experiences of consultations with physician associates in primary care in England: a qualitative study. Health Expect 2017;20:1011-9.

8 Department of Health. NHS Patient Experience Framework. London: NHS, 2012.

9 Hooker RS, Cipher DJ, Sekscenski E. Patient satisfaction with physician assistant, nurse practitioner, and physician care: a national survey of Medicare beneficiaries. J Clin Outcome Manag 2005;12:88-92.

10 Henry LR, Hooker RS. Retention of physician assistants in rural health clinics. J Rural Health 2007:23:207-14.

11 Berry LL, Seiders K, Grewal D. Understanding service convenience. J Market 2002:66:1-17.

12 Lin CT, Albertson GA, Schilling LM et al. Is patients' perception of time spent with the physician a determinant of ambulatory patient satisfaction? Arch Intern Med 2001:161:1437-42.

13 Ford RC, Bach SA, Fottler MD. Methods of measuring patient satisfaction in health care organizations. Health Care Manage Rev 1997;22:74-89.
14 Bachman JW. The problem with patient satisfaction scores. Fam Pract Manag 2016;23:23-7.

15 Fenton J], Jerant AF, Bertakis KD, Franks P. The cost of satisfaction: a national study of patient satisfaction, health care utilization, expenditures, and mortality. Arch Intern Med 2012;172:405-11.

16 Kravitz RL, Bell RA, Azari R et al. Request fulfillment in office practice: antecedents and relationship to outcomes. Med Care 2002:40:38-51.

17 Tsianakas V, Maben J, Wiseman T et al. Using patients' experiences to identify priorities for quality improvement in breast cancer care: patient narratives, surveys or both? BMC Health Serv Res 2012;12:271

Address for correspondence: Dr Qazi Zaman, George Eliot Hospital NHS Trust, Acute Medical Unit, College Street, Nuneaton CV10 7DJ, UK.

Email: qzaman99@aol.com

\section{NGC ${ }^{\text {noumes }}$

\section{New to systematic reviewing, or need} to improve your critical appraisal skills?

Our intensive 1-day courses will introduce you to the key principles of critical appraisal and systematic reviewing, and equip you with the basic skills to put your knowledge into practice.

$>$ Introduction to critical appraisal > Systematic reviews and meta-analysis in action

Discount available if both courses are booked together.

\section{'Informative and practical'} Dr Rajeswari Ramaraj

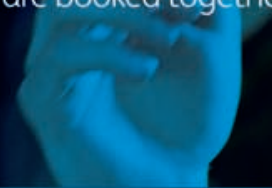

